\title{
Huge Ovarian Cyst Torsion: A Case Report
}

Sunita Maharjan, Ganesh Dangal, Aruna Karki, Hema Kumari Pradhan, Ranjana Shrestha, Kabin Bhattachan, Nishma Bajracharya, Kenusha Devi Tiwari, Sonu Bharati

\section{Author Info:}

Department of Obstetrics and Gynecology, Kathmandu Model Hospital, Kathmandu, Nepal

\section{Corresponding Author:}

Dr. Sunita Maharjan, Department of Obstetrics and Gynaecology, Kathmandu Model Hospital, Kathmandu, Nepal

\section{Email/Contact:}

sunitamaharjan811@gmail. com

Orchid ID: https://orcid. org/0000-0003-009-0092

\begin{abstract}
Ovarian cyst torsion (also termed as adnexal torsion) refers to partial or complete rotation of the ovary and a portion of fallopian tube along with its supply to vascular pedicle. It occurs commonly in females of all age group, more on the right side (60\%) and often with acute lower abdominal pain lasting for few hours up to 24 hours. Ovarian cyst torsion is one of the devastating conditions hampering blood supply of ovary which may lead to total necrosis of ovarian tissue like in our case. Delayed diagnosis and management can lead to various complications. Here, we present a case of 46 years old perimenopausal female, para 2, living 2 who presented to our emergency department with complain of sudden onset severe pain abdomen since morning. Emergency exploratory laparotomy was done due to high clinical suspicion of torsion based on previous ultrasound finding of ovarian cyst done a day prior to the presentation. On intraoperative finding, the cyst was already necrosed. Due to timely diagnosis and management by emergency laparotomy, anticipated complications were reduced.
\end{abstract}

Keywords: Necrosis, Laparotomy, Ovarian cyst, Ovarian torsion

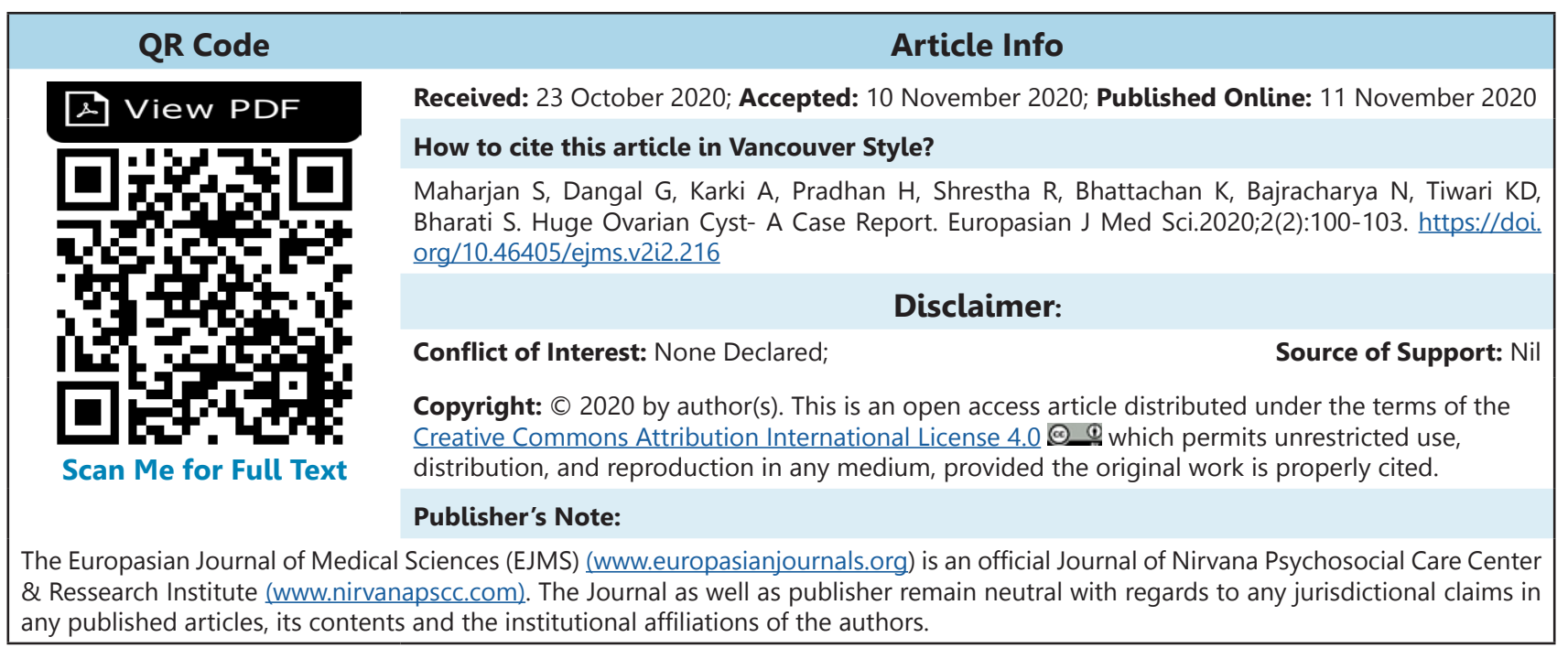




\section{INTRODUCTION}

Ovarian torsion refers to partial or complete rotation of ovary and a portion of fallopian tube along its supplying vascular pedicle. It is the fifth most common gynaecologic emergency surgery. ${ }^{1}$ It occurs commonly in reproductive age group, more on the right side (60\%) and often presents with lower abdominal pain lasting for few hours and upto 24 hours, accounting for $2.7 \%$ of acute gynecological conditions. ${ }^{2}$ Too often the physical signs are not specific and the final diagnosis as determined through laparoscopy or during laparotomy differs from that based on clinical grounds. ${ }^{3}$ It is an emergency condition requiring immediate surgery to salvage the ovary. Congenitally long ovarian ligaments, excessive laxity of the pelvic ligaments or a relatively small uterus that allows more space for the adnexa to twist on its axis may be predisposing factors. ${ }^{1}$ The infundibulopelvic ligaments suspend the movable ovary, allowing the ovary to position laterally and posteriorly to the uterus. The ovarian vessels travel along the infundibulopelvic ligaments which attach to the pelvic sidewall. Because adnexal tissue is not fixed, a big leading point, such as tumorous growth, can induce twisting. The medial side of the ovary is connected to the uterus by the uteroovarian ligament which is composed of muscular and fibrous tissue. Its function is to connect ovary to the uterus and support it. It also supplies blood from uterine artery to the ovary.

\section{CASE PRESENTATION}

A 46 years old multiparous perimenopausal female presented at our Emergency department with complain of abdominal pain which was generalized, more on the left lower quadrant, cramping type, severe in intensity since morning. Her last menstrual period was on 3 weeks prior to presentation. and it was a regular cycle. Bowel and bladder habits were normal. Past medical or surgical history were not significant with use of any contraception.

On presentation, the patient was conscious and well oriented with stable vital signs. On physical examination, she was obese. Cardinal signs absent. On systemic examination, her respiratory and cardiovascular findings were unremarkable. However, on per abdomen examination, a firm, freely mobile 18 weeks size mass was palpable on the right side of abdomen with tenderness and rebound tenderness present on the left iliac fossa. Speculum examination was not significant.

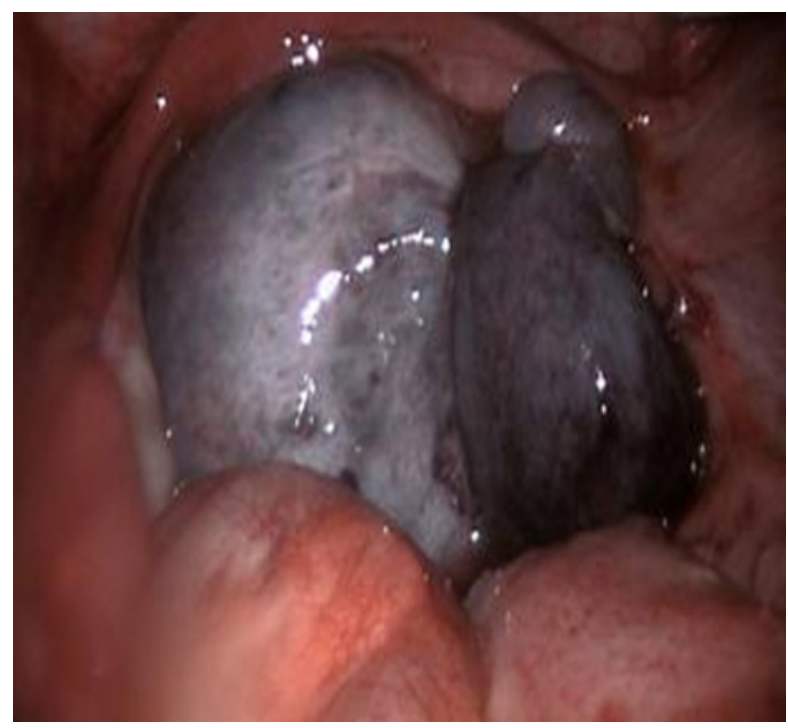

Figure 1: Intra-operative finding of huge ovarian cyst torsion

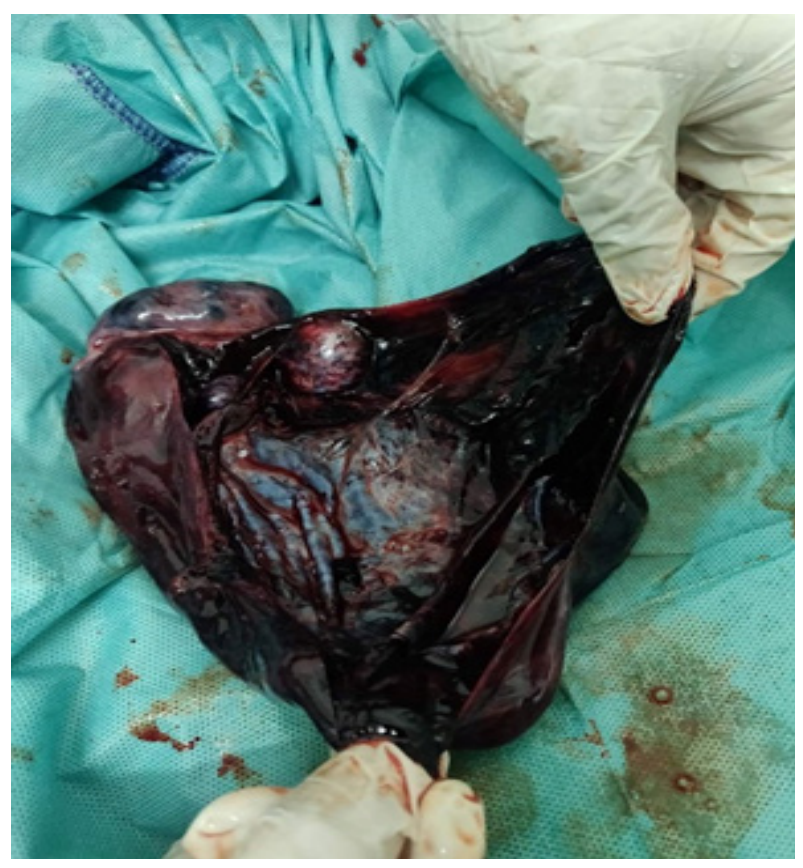

Figure 2: Cut-section of the cyst

On per vaginal examination, uterus normal size, anteverted, however, a tender cystic mass of approximately $11 \mathrm{~cm} \times 15 \mathrm{~cm}$ felt through pouch of Douglas mobile from side by side, tenderness present on cervical excitation. She was managed with NSAIDS for pain with some relief. Her urine pregnancy test was negative. Emergency ultrasound was done that showed large left adnexal cyst measuring $15 \times 11.4 \mathrm{~cm}$ and enlarged right ovary. Tumor markers like CA125 and CEA which was done after her finding of ovarian cyst on 
ultrasound a day prior to presentation were within normal limits. Informed consent was taken from the patient and patient party for the emergency laparotomy. Emergency laparotomy with left salpingo-oophorectomy was done on the same day with intraoperative findings of Ascitic fluid with blood stained, $15 \times 18 \mathrm{~cm}$ left ovarian cyst with necrotic appearance of cyst wall and ovary twisted two times. Serous type clear fluid approximately 1.5litres aspirated from the cyst. Right ovary and tube were normal. Postoperatively, patient was symptomatically relieved. Histopathology was suggestive of twisted serous cystadenoma. She was discharged on third post-operative day without any complications.

\section{DISCUSSION}

Discussion Ovarian torsion occurs due to partial or complete rotation of ovary and a portion of fallopian tube along its supplying vascular pedicle, hampering vascular supply of the ovary. Risk factors for ovarian torsion include pregnancy, ovarian stimulation, previous abdominal surgery, and tubal ligation. ${ }^{4}$ However, torsion is frequently associated with ovarian pathologies that result in enlarged ovaries. The most frequently encountered pathology is that of an ovarian dermoid, although other structures include parameso/tubal cysts, follicular cysts, endometriomas and serous/ mucinous cystadenoma. ${ }^{5}$

Data regarding cyst size and risk of torsion are conflicting, with some suggesting that torsion may be more likely in larger cysts $(>5 \mathrm{~cm}),{ }^{4}$ and others suggesting that cysts larger than $5 \mathrm{~cm}$ in size are less likely to undergo torsion than smaller ones and can be managed conservatively. ${ }^{6}$ As stated in the case report done by Nasirietal, ${ }_{1}^{7}$ cyst more than $10 \mathrm{~cm}$ is usually resected due to increased risk of malignancy, rupture or torsion. Management of cysts between 5 to $10 \mathrm{~cm}$ is controversial. ${ }^{7}$ Similarly, in this case, the size of the cyst was larger than $5 \mathrm{~cm}(15 \mathrm{~cm} \times 18$ $\mathrm{cm}$ ) and patient had already completed her family, she was treated by salpingo-oophorectomy. Sixtyfour percent of torsions occur on the right side. ${ }^{2}$ It is believed that the lower rate of torsion on the left side is attributed to the protective nature of the descending colon and shorter utero-ovarian ligament on this side compared to its counterpart. This finding has been consistent with a case study of a 22 -year-old nulliparous done by Cindy et al. ${ }^{8}$ In contrast to this, the torsion in our study occurred in the left side while the right tube and ovary was normal, which may have been aggravated by the size of the cyst.

Although the viability of an ovary declines as time elapses from the onset of pain to surgical detorsion, the ovary's dual blood supply makes it resilient to vascular injury and the exact duration of vascular interruption needed to cause irreversible damage to the ovary is unknown. If ovarian torsion is suspected, timely intervention with diagnostic laparoscopy is indicated to preserve ovarian function and future fertility. A surgeon should not remove a torsed ovary unless oophorectomy is unavoidable, such as when a severely necrotic ovary falls apart. The appearance of the ovary at time of surgery is not a reliable indicator of ovarian viability. Multiple studies report future ovarian function despite a grossly ischemic appearance at time of surgery.

Rody et $\mathrm{al}^{5}$ suggest conservative management of ovarian torsion regardless of the macroscopic appearance of the ovary. Their large literature review reported no severe complications, such as embolism or infection, even after the detorsion of "necrotic-looking" ovaries. In support of this, animal studies suggest that reperfusion of ischaemic ovaries even after 24 hours, with a time limiting interval of 36 hours, results in ovarian viability as demonstrated histologically. ${ }^{9}$

A study done by Balasubramaniam et $a{ }^{10}$ proved that duration of pain cannot predict the ovarian salvageability and laparoscopic detorsion with or without oophoropexy would be the treatment of choice for adnexal torsion irrespective of the grade of ischemia in females younger than 40 years old. However, the role of oophoropexy after detorsion to reduce the recurrence is not clear. However, the impact of oophoropexy on subsequent fertility merits further study.

Hence, there are many ways to perform the surgery and detorsion and ovarian conservation are almost always recommended now rather than salpingooophorectomy. An ovarian cystectomy is often performed for a benign ovarian mass. If malignancy is highly suspected, a salpingo-oophorectomy is needed.

\section{CONCLUSION}

Although the diagnosis of ovarian torsion is difficult and challenging, careful analysis of presenting symptoms such as sudden onset abdominal pain is very critical. It is apparent that prompt diagnosis is dependent on clinical history and a high index of suspicion. Accurate and detailed history taking is 
highly important, both of the presenting complaint and of the previous gynecological and surgical history. Physical examination may elicit an adnexal mass or adnexal tenderness but can be non-specific. Pelvic ultrasonography can provide information on ovarian cysts. Once ovarian torsion is suspected, surgery is the mainstay of diagnosis and treatment. Ovarian cystectomy, oophorectomy or conservative treatment with detorsion can be the treatment of choice.

\section{REFERENCES}

1. Abraham M, Keyser EA. Adnexal Torsion in Adolescents. Obstetrics and Gynecology. 2019;134(2):E56-63. $\quad$ https://doi.org/10.1097/ AOG.0000000000003373

2. Mishra VV, Nanda S, Nawal R, Choudhary S. Unusual presentation of twisted ovarian cyst. J Mi-life Health. 2016;7:31-3, IP: 27.34.20.157 https://doi. org/10.4103/0976-7800.179174

3. Anteby SO, Schenker JG, Polishuk WZ. The value of laparoscopy in acute pelvic pain. Ann Surg 1975;181(4):484-6. $\quad$ https://doi.org/10.1002/ bjs. 1800731230

4. Huchon C, Staraci S, Fauconnier A. Adnexal torsion: a predictive score for pre-operative diagnosis. Hum Reprod.2010;25(9):2276-80.https://doi.org/10.1093/ humrep/deq173

5. Rody A, Jackisch C, Klockenbusch W, Heinig J, Coenen-Worch V, Schneider HP. The conservative management of adnexal torsion- a case-report and review of the literature. Eur J Obstet Gynecol Reprod Biol. 2002;101:83-6. https://doi.org/10.1016/S03012115(01)00518-8

6. Kanizsai B, Örley J, Szigetvari I, Doszpod J. Ovarian cysts in children and adolescents: their occurrence, behavior, and management. Journal of pediatric and adolescent gynecology. 1998;11(2):85-8. https://doi. org/10.1016/S1083-3188(98)70117-2

7. Nasiri A, Rahimi S, Tomlinson E. Ovarian Torsion in Pregnancy: A Case Report. Gynecol Obstet Case Rep. 2017;3:2. https://doi.org/10.21767/2471-8165.1000051

8. Chen $\mathrm{HE}$, Georgiou C. Ovarian torsion in a 22-year old nulliparous woman. AMSJ. 2012;3 (1):58-60. https://ro.uow.edu.au/ihmri/369

9. Taskin O, Birincioglu M, Aydin A, Buhur A, Burak F, Yilmaz $I$, et al. The effect of twisted ischemic adnexa managed by detorsion on ovarian viability and histology: an ischemia reperfusion rodent model. Hum Reprod. 1998;13(10):2823-7 https://doi. org/10.1093/humrep/13.10.2823.

10. Balasubramaniam D, Duraisamy KY, Ezhilmani M. Laparoscopic Detorsion and Fertility Preservation in Twisted Ischemic Adnexa-A Single-Center Prospective Study. Gynecol Minim Invasive Ther. 2020;9(1):24. https://doi.org/10.4103/GMIT. GMIT 2019 